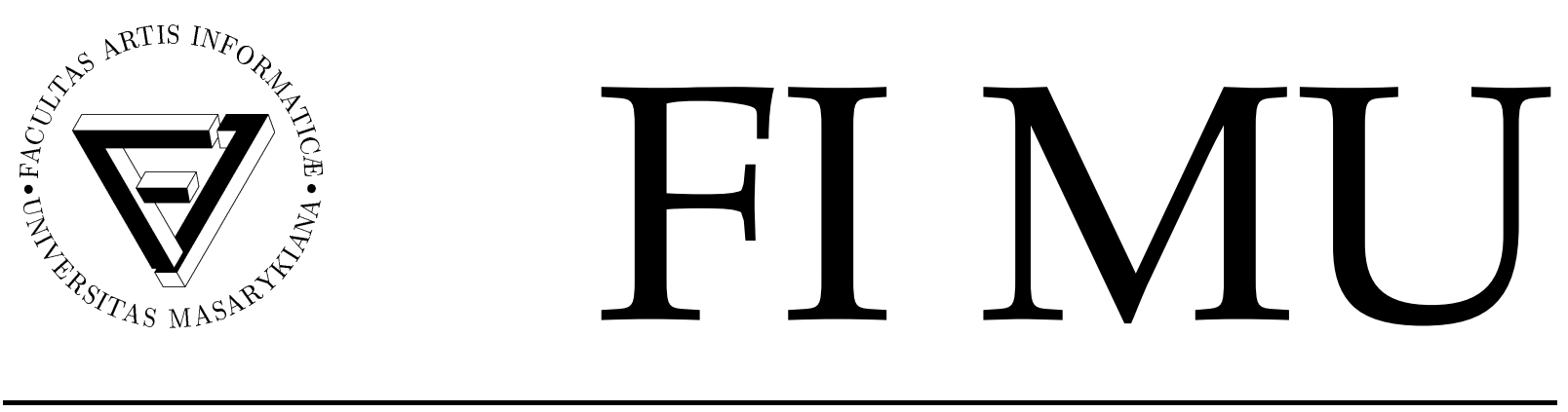

Faculty of Informatics Masaryk University Brno

\title{
Checking Thorough Refinement on Modal Transition Systems Is EXPTIME-Complete
}

by

\author{
Nikola Beneš \\ Jan Křetínský \\ Kim G. Larsen \\ Jiří Srba
}

FI MU Report Series

FIMU-RS-2009-03

Copyright (C) 2009, FI MU

July 2009 
Copyright (C) 2009, Faculty of Informatics, Masaryk University. All rights reserved.

Reproduction of all or part of this work is permitted for educational or research use on condition that this copyright notice is included in any copy.

Publications in the FI MU Report Series are in general accessible via WWW:

http://www.fi.muni.cz/reports/

Further information can be obtained by contacting:

Faculty of Informatics

Masaryk University

Botanická 68a

60200 Brno

Czech Republic 


\title{
Checking Thorough Refinement on Modal Transition Systems Is EXPTIME-Complete
}

\author{
Nikola Beneš \\ Jan Křetínský ${ }^{\dagger}$ \\ Faculty of Informatics, Masaryk University, \\ Botanická 68a, 60200 Brno, Czech Republic \\ \{xbenes3,xkretins\}@fi.muni.cz \\ Kim G. Larsen \\ Jiř́i Srba ${ }^{\S}$ \\ Department of Computer Science, Aalborg University, \\ Selma Lagerlöfs Vej 300, 9220 Aalborg Øst, Denmark \\ $\{k g l$, srba\}@cs . aau.dk
}

July 28, 2009

\begin{abstract}
Modal transition systems (MTS), a specification formalism introduced more than 20 years ago, has recently received a considerable attention in several different areas. Many of the fundamental questions related to MTSs have already been answered. However, the problem of the exact computational complexity of thorough refinement checking between two finite MTSs remained unsolved.

We settle down this question by showing EXPTIME-completeness of thorough refinement checking on finite MTSs. The upper-bound result relies on a novel algorithm running in single exponential time providing a direct goal-oriented way to decide thorough refinement. If the right-hand side MTS is moreover deterministic,
\end{abstract}

*Partially supported by the Academy of Sciences of the Czech Republic, project No. 1ET408050503.

${ }^{\dagger}$ Partially supported by the research centre ITI, project No. 1M0545.

†Partially supported by the VKR Center of Excellence MT-LAB.

\$ Partially supported by Ministry of Education of the Czech Republic, project No. MSM 0021622419. 
or has a fixed size, the running time of the algorithm becomes polynomial. The lower-bound proof is achieved by reduction from the acceptance problem of alternating linear bounded automata and the problem remains EXPTIME-hard even if the left-hand side MTS is fixed.

\section{Introduction}

Modal transition systems (MTS) is a specification formalism which extends the standard labelled transition systems with two types of transitions, the may transitions that are allowed to be present in an implementation of a given modal transition system and must transitions that must be necessarily present in any implementation. Modal transition systems hence allow to specify both safety and liveness properties. The MTS framework was suggested more than 20 years ago by Larsen and Thomsen [15] and has recently brought a considerable attention due to several applications to e.g. componentbased software development [17,6], interface theories [21, 18], modal abstractions and program analysis $[10,13,16]$ and other areas $[9,22]$, just to mention a few of them. A renewed interest in tool support for modal transition systems is recently also emerging $[7,8]$. A recent overview article on the theoretical foundations of MTSs and early tool development is available in [1].

Modal transition systems were designed to support component-based system development via a stepwise refinement process where abstract specifications are gradually refined into more concrete ones until an implementation of the system (where the may and must transitions coincide) is obtained. One of the fundamental questions is the decidability of a thorough refinement relation between two specifications $S$ and $T$. We say that $S$ thoroughly refines $T$ iff every implementation of $S$ is also an implementation of $T$. While for a number of other problems, like the common implementation problem, a matching complexity lower and upper bounds were given $[2,14,3]$, the question of the exact complexity of thorough refinement checking between two finite MTSs remained unanswered.

In this paper, we prove EXPTIME-completeness of thorough refinement checking between two finite MTSs. The hardness result is achieved by a reduction from the acceptance problem of alternating linear bounded automata, a well known EXPTIMEcomplete problem, and it improves the previously established PSPACE-hardness [2]. The main reduction idea is based on the fact that the existence of a computation step 
between two configurations of a Turing machine can be locally verified (one needs to consider the relationships between three tape symbols in the first configuration and the corresponding three tape symbols in the second one, see e.g. [20, Theorem 7.37]), however, a nonstandard encoding of computations of Turing machines (which is crucial for our reduction) and the addition of the alternation required a nontrivial technical treatment. Moreover, we show that the problem remains EXPTIME-hard even if the lefthand side MTS is of a constant size. Some proof ideas for the containment in EXPTIME were mentioned in [2] where the authors suggest a reduction of the refinement problem to validity checking of vectorized modal $\mu$-calculus, which can be solved in EXPTIMEthe authors in [2] admit that such a reduction relies on an unpublished popular wisdom, and they only sketch the main ideas hinting at the EXPTIME algorithm. In our paper, we describe a novel technique for deciding thorough refinement in EXPTIME. The result is achieved by a direct goal-oriented algorithm performing a least fixed-point computation, and can be easily turned into a tableau-based algorithm. As a corollary, we also get that if the right-hand side MTS is deterministic (or of a constant size), the algorithm for solving the problem runs in deterministic polynomial time.

Details of some technical proofs were moved into the appendix.

\section{Basic Definitions}

A modal transition system (MTS) over an action alphabet $\Sigma$ is a triple $(\mathrm{P},--\rightarrow, \longrightarrow)$, where $\mathrm{P}$ is a set of processes and $\longrightarrow \subseteq-\rightarrow \subseteq \mathrm{P} \times \Sigma \times \mathrm{P}$ are must and may transition relations, respectively. The class of all MTSs is denoted by $\mathcal{M T S}$. Because in MTS whenever $S \stackrel{a}{\longrightarrow} S^{\prime}$ then necessarily also $S \stackrel{a}{\rightarrow} S^{\prime}$, we adopt the convention of drawing only the must transitions $S \stackrel{a}{\longrightarrow} S^{\prime}$ in such cases. An MTS is finite if $P$ and $\Sigma$ are finite sets.

An MTS is an implementation if $-\rightarrow=\longrightarrow$. The class of all implementations is denoted $i \mathcal{M T S}$ and as in implementations the must and may relations coincide, we can consider such systems as the standard labelled transition systems.

Definition 2.1. Let $M_{1}=\left(P_{1},-\rightarrow_{1}, \longrightarrow \longrightarrow_{1}\right), M_{2}=\left(P_{2},-\rightarrow_{2}, \longrightarrow_{2}\right)$ be MTSs over the same action alphabet $\Sigma$ and $\mathrm{S} \in \mathrm{P}_{1}, \mathrm{~T} \in \mathrm{P}_{2}$ be processes. We say that $\mathrm{S}$ modally refines $\mathrm{T}$, written $\mathrm{S} \leq_{\mathrm{m}} \mathrm{T}$, if there is a relation $\mathrm{R} \subseteq \mathrm{P}_{1} \times \mathrm{P}_{2}$ such that $(\mathrm{S}, \mathrm{T}) \in \mathrm{R}$ and for every $(\mathrm{A}, \mathrm{B}) \in \mathrm{R}$ and every $a \in \Sigma$ :

1. if $\mathrm{A} \stackrel{\mathrm{a}}{\rightarrow-\rightarrow} \mathrm{A}^{\prime}$ then there is a transition $\mathrm{B} \stackrel{\mathrm{a}}{\rightarrow-\rightarrow_{2}} \mathrm{~B}^{\prime}$ s.t. $\left(\mathrm{A}^{\prime}, \mathrm{B}^{\prime}\right) \in R$, and 

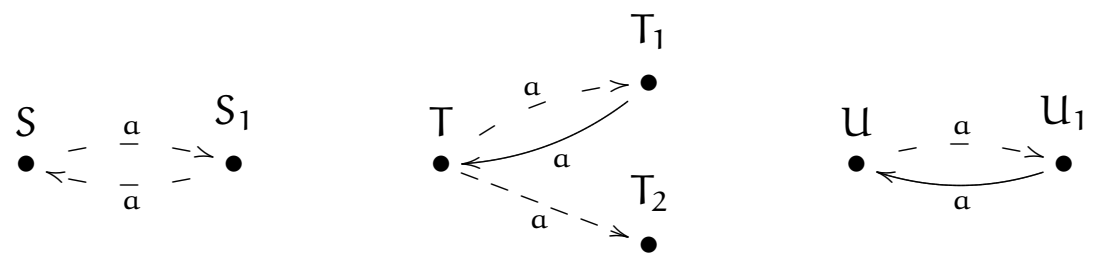

Figure 1: $S \leq_{t} T$ but $S \not \leq_{m} T$, and $S \not \underline{Z}_{t} U$ and $S \not \underline{Z}_{m} U$

2. if $\mathrm{B} \stackrel{\mathrm{a}}{\longrightarrow} 2 \mathrm{~B}^{\prime}$ then there is a transition $\mathrm{A} \stackrel{\mathrm{a}}{\longrightarrow} \mathrm{A}^{\prime}$ s.t. $\left(\mathrm{A}^{\prime}, \mathrm{B}^{\prime}\right) \in R$.

We often omit the indices in the transition relations and use symbols $-\rightarrow$ and $\longrightarrow$ whenever it is clear from the context what transition system we have in mind. Note that on implementations modal refinement coincides with the classical notion of strong bisimilarity, and on modal transition systems without any must transitions it corresponds to the well-studied simulation preorder.

Example 2.2. Consider processes $\mathrm{S}$ and $\mathrm{T}$ in Fig. 1. We prove that $\mathrm{S}$ does not modally refine $\mathrm{T}$. Indeed, there is a may-transition $\mathrm{S} \stackrel{\mathrm{a}}{\rightarrow} \mathrm{S}_{1}$ on the left-hand side which has to be matched by entering either $\mathrm{T}_{1}$ or $\mathrm{T}_{2}$ on the right-hand side. However, in the first case there is a move $\mathrm{T}_{1} \stackrel{\mathrm{a}}{\longrightarrow} \mathrm{T}$ on the right-hand side which cannot be matched from $\mathrm{S}_{1}$ as it has no must-transition under $a$. In the second case there is a may-transition $S_{1} \stackrel{a}{\rightarrow} S$ on the left-hand side which cannot be matched by any may-transition from $\mathrm{T}_{2}$. Hence there cannot be any relation of modal refinement containing the pair $\mathrm{S}$ and $\mathrm{T}$, which means that $\mathrm{S} \mathbb{Z}_{\mathrm{m}} \mathrm{T}$. Similarly, one can argue that $\mathrm{S} \not \underline{\mathrm{Z}}_{\mathrm{m}} \mathrm{U}$.

We shall now observe that the modal refinement problem, i.e. the question whether a given process modally refines another given process, is tractable for finite MTSs.

Theorem 2.3. The modal refinement problem for finite MTSs is P-complete.

Proof. Modal refinement can be computed in polynomial time by the standard greatest fixed-point computation, similarly as in the case of strong bisimulation. P-hardness of modal refinement follows from P-hardness of bisimulation [4] (see also [19]).

We proceed with the definition of thorough refinement, a relation that holds for two modal specification $S$ and $T$ iff any implementation of $S$ is also an implementation of $T$.

Definition 2.4. For a process $S$ let us denote by $\llbracket S \rrbracket=\left\{I \in i \mathcal{M T S} \mid \mathrm{I} \leq_{m}\right.$ S $\}$ the set of all implementations of $\mathrm{S}$. We say that $\mathrm{S}$ thoroughly refines $\mathrm{T}$, written $\mathrm{S} \leq_{\mathrm{t}} \mathrm{T}$, if $\llbracket \mathrm{S} \rrbracket \subseteq \llbracket \mathrm{T} \rrbracket$. 
Clearly, if $S \leq_{m} T$ then also $S \leq_{t}$ T because the relation $\leq_{m}$ is transitive. The opposite implication, however, does not hold as demonstrated by the processes $S$ and $\mathrm{T}$ in Fig. 1 where one can easily argue that every implementation of $S$ is also an implementation of $\mathrm{T}$. On the other hand, $\mathrm{S} \mathbb{Z}_{\mathrm{t}} \mathrm{U}$ because a process with just a single a-transition is an implementation of $S$ but not of $U$.

\section{Thorough Refinement Is EXPTIME-Hard}

In this section we prove that the thorough refinement relation $\leq_{\mathrm{t}}$ on finite modal transition systems is EXPTIME-hard by reduction from the acceptance problem of alternating linear bounded automata.

\subsection{Alternating Linear Bounded Automata}

Definition 3.1. An alternating linear bounded automaton (ALBA) is a tuple $\mathcal{M}=\left(\mathrm{Q}, \mathrm{Q}_{\forall}\right.$, $\left.\mathrm{Q}_{\exists}, \Sigma, \Gamma, \mathrm{q}_{\mathrm{O}}, \mathrm{q}_{\mathrm{acc}}, \mathrm{q}_{\mathrm{rej}}, \vdash,-\dashv, \delta\right)$ where $\mathrm{Q}$ is a finite set of control states partitioned into $\mathrm{Q}_{\forall}$ and $\mathrm{Q}_{\exists}$, universal and existential states, respectively, $\Sigma$ is a finite input alphabet, $\Gamma \supseteq \Sigma$ is a finite tape alphabet, $\mathrm{q}_{\mathrm{o}} \in \mathrm{Q}$ is the initial control state, $\mathrm{q}_{\mathrm{acc}} \in \mathrm{Q}$ is the accepting state, $\mathrm{q}_{\mathrm{rej}} \in \mathrm{Q}$ is the rejecting state, $\vdash, \dashv \in \Gamma$ are the left-end and the right-end markers that cannot be overwitten or moved, and $\delta:\left(\mathrm{Q} \backslash\left\{\mathrm{q}_{\mathrm{acc}}, \mathrm{q}_{\mathrm{re} j}\right\}\right) \times \Gamma \rightarrow 2^{\mathrm{Q} \times \Gamma \times\{\mathrm{L}, \mathrm{R}\}}$ is a computation step function such that for all $\mathbf{q}, \mathrm{p} \in \mathrm{Q}$ if $\delta(\mathrm{q}, \vdash) \ni(\mathrm{p}, \mathrm{a}, \mathrm{D})$ then $\mathrm{a}=\vdash, \mathrm{D}=\mathrm{R}$; if $\delta(\mathrm{q}, \dashv) \ni(\mathrm{p}, \mathrm{a}, \mathrm{D})$ then $\mathrm{a}=\dashv, \mathrm{D}=\mathrm{L} ;$ if $\delta(\mathrm{q}, \mathrm{a}) \ni(\mathrm{p}, \vdash, \mathrm{D})$ then $\mathrm{a}=\vdash$; and if $\delta(\mathrm{q}, \mathrm{a}) \ni(\mathrm{p}, \dashv, \mathrm{D})$ then $\mathrm{a}=\dashv$.

Remark 3.2. W.l.o.g. we assume that $\Sigma=\{a, b\}, \Gamma=\{a, b, \vdash, \dashv\}, Q \cap \Gamma=\emptyset$ and that for each $\mathrm{q} \in \mathrm{Q}_{\forall}$ and $\mathrm{a} \in \Gamma$ it holds that $\delta(\mathrm{q}, \mathrm{a})$ has exactly two elements $\left(\mathrm{q}_{1}, \mathrm{a}_{1}, \mathrm{D}_{1}\right),\left(\mathrm{q}_{2}, \mathrm{a}_{2}, \mathrm{D}_{2}\right)$ where moreover $\mathrm{a}_{1}=\mathrm{a}_{2}$ and $\mathrm{D}_{1}=\mathrm{D}_{2}$. We fix this ordering and the successor states $\mathrm{q}_{1}$ and $\mathrm{q}_{2}$ are referred to as the first and the second successor, respectively. The states $\mathrm{q}_{\mathrm{acc}}, \mathrm{q}_{\mathrm{rej}}$ have no successors.

A configuration of $\mathcal{M}$ is given by the state, the position of the head and the content of the tape. For technical reasons, we write it as a word over the alphabet $\Xi=\mathrm{Q} \cup$ $\Gamma \cup\{\vdash, \dashv, \exists, \forall, 1,2, *\}$ (where $\exists, \forall, 1,2, *$ are fresh symbols) in the following way. If the tape contains a word $\vdash w_{1} a w_{2} \dashv$, where $w_{1}, w_{2} \in \Gamma^{*}$ and $a \in \Gamma$, and the head is scanning the symbol $a$ in a state $q$, we write the configuration as $\vdash w_{1} \alpha \beta q a w_{2} \dashv$ where $\alpha \beta \in$ $\{\exists *, \forall 1, \forall 2\}$. 
The two symbols $\alpha \beta$ before the control state in every configuration are nonstandard, though important for the encoding of the computations into modal transition systems to be checked for thorough refinement. Intuitively, if a control state $q$ is preceded by $\forall 1$ then it signals that the previous configuration (in a given computation) contained a universal control state and the first successor was chosen; similarly $\forall 2$ reflects that the second successor was chosen. Finally, if the control state is preceded by $\exists *$ then the previous control state was existential and in this case we do not keep track of which successor it was, hence the symbol $*$ is used instead. The initial configuration for an input word $w$ is by definition $\vdash \exists * q_{0} w \dashv$.

Depending on the present control state, every configuration is called either universal, existential, accepting or rejecting.

A step of computation is a relation $\rightarrow$ between configurations defined as follows (where $w_{1}, w_{2} \in \Gamma^{*}, \alpha \beta \in\{\forall 1, \forall 2, \exists *\}, a, b, c \in \Gamma, i \in\{1,2\}$, and $w_{1} a w_{2}$ and $w_{1} c a w_{2}$ both begin with $\vdash$ and end with $\dashv$ ):

- $w_{1} \alpha \beta q a w_{2} \rightarrow w_{1} b \forall i p w_{2}$ if $\delta(q, a) \ni(p, b, R), q \in Q_{\forall}$ and $(p, b, R)$ is the $i^{\prime}$ th successor,

- $w_{1} \alpha \beta \mathrm{qa} w_{2} \rightarrow w_{1} \mathrm{~b} \exists * \mathrm{p} w_{2}$ if $\delta(q, a) \ni(p, b, R)$ and $q \in Q_{\exists}$,

- $w_{1} c \alpha \beta q a w_{2} \rightarrow w_{1} \forall i p c b w_{2}$ if $\delta(q, a) \ni(p, b, L), q \in Q_{\forall}$ and $(p, b, L)$ is the $i^{\prime}$ th successor, and

- $w_{1} c \alpha \beta q a w_{2} \rightarrow w_{1} \exists * p c b w_{2}$ if $\delta(q, a) \ni(p, b, L)$ and $q \in Q_{\exists}$.

Note that for an input $w$ of length $n$ all reachable configurations are of length $n+5$. A standard result is that one can efficiently compute the set Comp $\subseteq \Xi^{10}$ of all compatible 10-tuples such that for each sequence $C=c_{1} c_{2} \cdots c_{k}$ of configurations $c_{1}, c_{2}, \ldots, c_{k}$, with the length of the first configuration being $l=\left|c_{1}\right|=n+5$, we have $c_{1} \rightarrow c_{2} \rightarrow \cdots \rightarrow c_{k}$ iff for all $i, 0 \leq i \leq(k-1) l-5$,

$$
\begin{aligned}
& (C(i+1), C(i+2), C(i+3), C(i+4), C(i+5), \\
& C(i+1+l), C(i+2+l), C(i+3+l), C(i+4+l), C(i+5+l)) \in \text { Comp. }
\end{aligned}
$$

A computation tree for $\mathcal{M}$ on an input $w \in \Sigma^{*}$ is a tree $T$ satisfying the following: the root of $\mathrm{T}$ is (labeled by) the initial configuration, and whenever $\mathrm{N}$ is a node of $\mathrm{T}$ labeled by a configuration $\mathrm{c}$ then the following holds: 
- if $\mathrm{c}$ is accepting or rejecting then $\mathrm{N}$ is a leaf;

- if $\mathrm{c}$ is existential then $\mathrm{N}$ has one child labeled by some $\mathrm{d}$ such that $\mathrm{c} \rightarrow \mathrm{d}$;

- if $\mathrm{c}$ is universal then $\mathrm{N}$ has two children labelled by the first and the second successor of c, respectively.

Without loss of generality, we shall assume from now on that any computation tree for $\mathcal{M}$ on an input $w$ is finite (see e.g. [20, page 198]) and that every accepting configuration contains at least four other symbols following after the state $\mathrm{q}_{\mathrm{acc}}$.

We say that $\mathcal{M}$ accepts $w$ iff there is a (finite) computation tree for $\mathcal{M}$ on $w$ with all leaves labelled with accepting configurations. The following fact is well known (see e.g. [20]).

Proposition 3.3. Given an ALBA M and a word $w$, the problem whether $M$ accepts $w$ is EXPTIME-complete.

\subsection{Encoding of Configurations and Computation Trees}

In this subsection we shall discuss the particular encoding techniques necessary for showing the lower bound. For technical convenience we will consider only tree encodings and so we first introduce the notion of tree-thorough refinement.

Definition 3.4. Let Tree denote the class of all MTSs with their graphs being trees. We say that a process $S$ tree-thoroughly refines a process $T$, denoted by $S \leq_{\mathrm{tt}} \mathrm{T}$, if $\llbracket S \rrbracket \cap$ Tree $\subseteq \llbracket T \rrbracket \cap$ Tree.

Lemma 3.5. For any two processes $\mathrm{S}$ and $\mathrm{T}, \mathrm{S} \leq_{\mathrm{tt}} \mathrm{T}$ iff $\mathrm{S} \leq_{\mathrm{t}} \mathrm{T}$.

Proof. The if case is trivial. For the only if case, we define an unfold $\mathrm{U}(\mathrm{S})$ of a process $S$ over an MTS $M=(P,--\rightarrow, \longrightarrow)$ with an alphabet $\Sigma$ to be a process $S$ over an MTS $\mathrm{U}(\mathrm{M})=\left(\mathrm{P}^{*},-\rightarrow \mathrm{U}, \longrightarrow \mathrm{U}\right)$ over the same alphabet and where $\mathrm{P}^{*}$ is the set of all finite sequences over the symbols from $P$. The transition relations are defined as follows: for all $a \in \Sigma, T, R \in P$ and $\alpha \in P^{*}$, whenever $T \stackrel{a}{\rightarrow} R$ then $\alpha T_{-}^{\rightarrow} \rightarrow \mathrm{u} \alpha T R$, and whenever $\mathrm{T} \stackrel{\mathrm{a}}{\longrightarrow} \mathrm{R}$ then $\alpha \mathrm{T} \stackrel{\mathrm{a}}{\longrightarrow} \mathrm{u} \alpha \mathrm{TR}$. Since the transitions in $\mathrm{U}(\mathrm{S})$ depend only on the last symbol, we can easily see that $\mathrm{U}(\mathrm{S}) \leq_{\mathrm{m}} \mathrm{S}$ and $\mathrm{S} \leq_{\mathrm{m}} \mathrm{U}(\mathrm{S})$ for every process $\mathrm{S}$.

Let I be now an implementation of $S$. Its unfold $U(\mathrm{I})$ is also an implementation of $\mathrm{S}$ by $\mathrm{U}(\mathrm{I}) \leq_{\mathrm{m}} \mathrm{I} \leq_{\mathrm{m}} \mathrm{S}$ and the transitivity of $\leq_{\mathrm{m}}$. By our assumption that $\mathrm{S} \leq_{\mathrm{tt}} \mathrm{T}$ and the fact that $\mathrm{U}(\mathrm{I})$ is a tree, we get that $\mathrm{U}(\mathrm{I})$ is also an implementation of $\mathrm{T}$. Finally, $\mathrm{I} \leq_{\mathrm{m}}$ 
$\mathrm{U}(\mathrm{I}) \leq_{\mathrm{m}} \mathrm{T}$ and the transitivity of $\leq_{\mathrm{m}}$ allow us to conclude that $\mathrm{I}$ is an implementation of $\mathrm{T}$.

Let $\mathcal{M}=\left(\mathrm{Q}, \mathrm{Q}_{\forall}, \mathrm{Q}_{\exists}, \Sigma, \Gamma, \mathrm{q}_{0}, \mathrm{q}_{\mathrm{acc}}, \mathrm{q}_{\mathrm{rej}}, \vdash, \dashv, \delta\right)$ be an ALBA and $w \in \Sigma^{*}$ an input word of length $\mathrm{n}$. We shall construct (in polynomial time) modal transition systems $\mathrm{L}$ and $\mathrm{R}$ such that $\mathcal{M}$ accepts $w$ iff $L \not_{t t} R$. The system $L$ will encode (almost) all trees beginning with the initial configuration, while the implementations of R encode only the incorrect or rejecting computation trees.

Configurations, i.e. sequences of letters from $\Xi$, are not encoded straightforwardly as sequences of actions (the reason why this naive encoding does not work is explained later on in Remark 3.12). Instead we have to use two auxiliary actions $\pi$ a $\sigma$. The intended implementations of $L$ and $R$ will alternate between the actions $\pi$ and $\sigma$ on a linear path, while the symbols in the encoded configuration are present as side-branches on the path.

Formally, a sequence $a_{1} a_{2} a_{3} \cdots a_{n} \in \Xi^{*}$ is encoded as

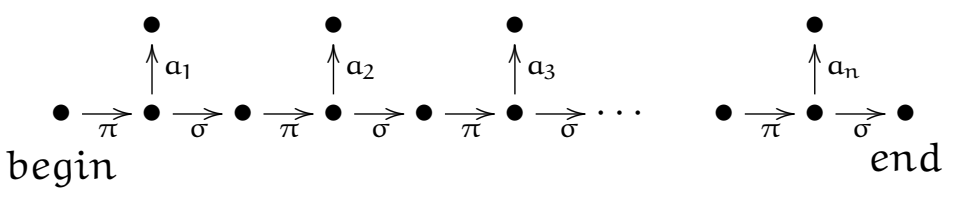

and denoted by code $\left(a_{1} a_{2} \cdots a_{n}\right)$.

We now describe how to transform computation trees into their corresponding implementations. We simply concatenate the subsequent codes of configurations in the computation tree such that the end node of the previous configuration is merged with the begin node of the successor configuration. Whenever there is a (universal) branching in the tree, we do not branch in the corresponding implementation at its beginning but we wait until we reach the occurrence of $\forall$. The branching happens exactly before the symbols 1 or 2 that follow after $\forall$. This occurs in the same place on the tape in both of the configurations due to the assumption that the first and the second successor move simultaneously either to the left or to the right, and write the same symbol (see Remark 3.2). A formal definition of the encoding of computation trees into implementations follows.

Definition 3.6 (Encoding computation trees into implementations). Let $\mathcal{T}$ be a (finite) computation tree. We define its tree implementation $\operatorname{code}(\mathcal{T})$ inductively as follows:

- if $\mathcal{T}$ is a leaf labelled with a configuration $\mathrm{c}$ then $\operatorname{code}(\mathcal{T})=\operatorname{code}(\mathrm{c})$; 


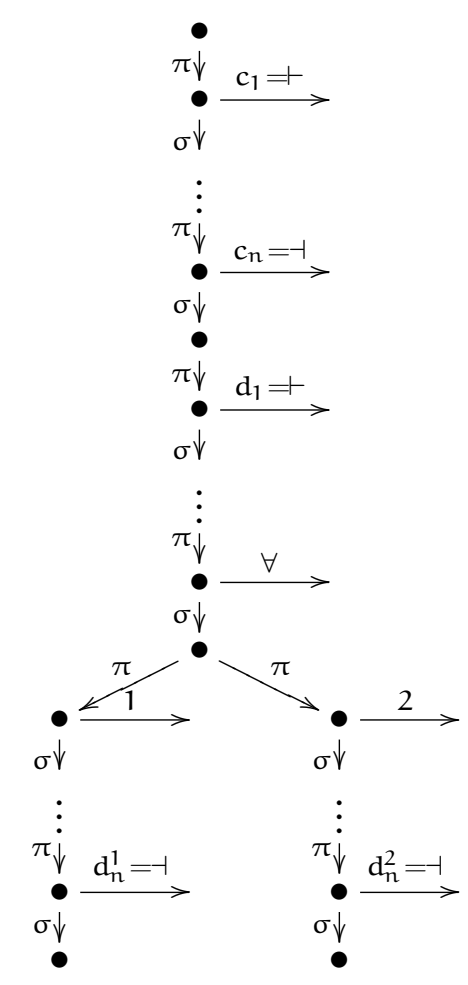

Figure 2: Comp. Tree Encoding

- if the root of $\mathcal{T}$ is labelled by an existential configuration c with a tree $\mathcal{T}^{\prime}$ being its child, then code $(\mathcal{T})$ is rooted in the begin node of code $(\mathrm{c})$, followed by code $\left(\mathcal{T}^{\prime}\right)$ where the end node of $\operatorname{code}(\mathrm{c})$ and the begin node of $\operatorname{code}\left(\mathcal{T}^{\prime}\right)$ are identified;

- if the root of $\mathcal{T}$ is labelled by a universal configuration c with two children $\mathrm{d}_{1} \ldots \forall 1 \ldots \mathrm{d}_{\mathrm{n}}^{1}$ and $\mathrm{d}_{1} \ldots \forall 2 \ldots \mathrm{d}_{\mathrm{n}}^{2}$ that are roots of the subtrees $\mathcal{T}_{1}$ and $\mathcal{T}_{2}$, respectively, then $\operatorname{code}(\mathcal{T})$ is rooted in the begin node of code $(\mathrm{c})$, followed by two subtrees code $\left(\mathcal{T}_{1}\right)$ and $\operatorname{code}\left(\mathcal{T}_{2}\right)$ where the nodes in code $\left(\mathrm{d}_{1} \ldots \forall\right)$ of the initial part of $\operatorname{code}\left(\mathcal{T}_{1}\right)$ are identified with the corresponding nodes in the initial part of $\operatorname{code}\left(\mathcal{T}_{2}\right)$ (note that by Remark 3.2 this prefix is common in both subtrees), and finally the end node of code(c) is identified with now the common begin node of both subtrees.

Fig. 2 illustrates this definition on a part of a computation tree, where the first configuration $c_{1} \ldots c_{n}$ is universal and has two successor configurations $d_{1} \ldots \forall 1 \ldots d_{n}^{1}$ and $\mathrm{d}_{1} \ldots \forall 2 \ldots \mathrm{d}_{\mathrm{n}}^{2}$. 


\subsection{The Reduction-Part 1}

We now proceed with the reduction. As mentioned earlier, our aim is to construct for a given ALBA $\mathcal{M}$ and a string $w$ two modal transition systems $L$ and $R$ such that $L \mathbb{E}_{\mathrm{tt}} \mathrm{R}$ iff $\mathcal{M}$ accepts $w$. Implementations of $L$ will include all (also incorrect) possible computation trees. We only require that they start with the encoding of the initial configuration and do not "cheat" in the universal branching (i.e. after the encoding of every symbol $\forall$ there must follow a branching such that at least one of the branches encodes the symbol 1 and at least another one encodes the symbol 2).

As $L$ should capture implementations corresponding to computations starting in the initial configuration, we set $L$ to be the begin of $\operatorname{code}\left(\vdash \exists * q_{0} w-1\right)$ and denote its end by M. After the initial configuration has been forced, we allow all possible continuations of the computation. This can be simply done by setting

$$
\begin{aligned}
& M_{\stackrel{\pi}{\rightarrow}}^{\rightarrow} M_{a} \\
& M_{a} \stackrel{\sigma}{\longrightarrow} M \\
& M_{a} \stackrel{a}{\longrightarrow} X_{a}
\end{aligned}
$$

for all letters $a \in \Xi \backslash\{\forall\}$ and there are no outgoing transitions from $X_{a}$.

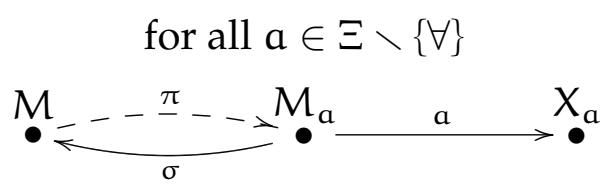

Finally, we add a fragment of MTS into the constructed process L which will guarantee the universal branching as mentioned above whenever the symbol $\forall$ occurs on a sidebranch. The complete modal transition system $L$ is now depicted in Fig. 3.

We shall now state some simple observations regarding tree implementations of the process L.

Proposition 3.7. Every tree implementation I of the process L satisfies that

1. every branch in $\mathrm{I}$ is labelled by an alternating sequence of $\pi$ and $\sigma$ actions, beginning with the action $\pi$, and if the branch is finite then it ends either with the action $\sigma$ or with an actions $a \in \Xi \backslash\{\forall\}$, and

2. every state in I with an incoming transition under the action $\pi$ has at least one outgoing transition under the action $\sigma$ and at least one outgoing transition under an action $a \in \Xi$, and 
$\underbrace{\text { code of the initial configuration }}$

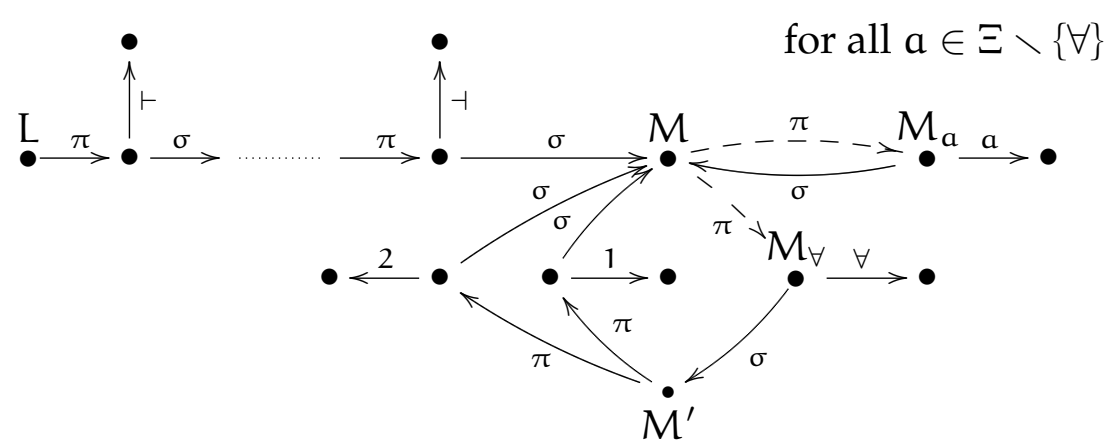

Figure 3: Full specification of the process L

3. whenever from any state in I there are two outgoing transitions under some $\mathrm{a} \in \Xi$ and $\mathrm{b} \in \Xi$ then $\mathrm{a}=\mathrm{b}$, and moreover no further actions are possible after taking any transition under $\mathrm{a} \in \Xi$, and

4. every branch in I longer than $2(n+5)$ begins with the encoding of the initial configuration $\vdash \exists * \mathrm{q}_{0} w \dashv$ where $\mathrm{n}=|w|$, and

5. every state in I with an incoming transition under $\sigma$ from a state where the action $\forall$ is enabled satisfies that every outgoing transition under $\pi$ leads to a state where either the action 1 or 2 is enabled (but not both at the same time), and moreover it has at least one such transition that enables the action 1 and at least one that enables the action 2 .

Of course, not every tree implementation of the process L represents a correct computation tree of the given ALBA. Implementations of L can widely (even uncountably) branch at any point and sequences of configurations they encode on some (or all) of their branches may not be correct computations of the given ALBA. Nevertheless, the encoding of any computation tree of the given ALBA is an implementation of the processes $\mathrm{L}$, as stated by the following lemma.

Lemma 3.8. Let $\mathrm{T}$ be a computation tree of an $A L B A \mathcal{M}$ on an input $w$. Then $\operatorname{code}(\mathrm{T}) \leq_{\mathrm{m}} \mathrm{L}$. Proof sketch. To show that the implementation $\operatorname{code}(\mathrm{T})$ modally refines $\mathrm{L}$ is rather straightforward. The implementation code $(\mathrm{T})$ surely starts with the encoding of the initial configuration and all symbols $a \in \Xi \backslash\{\forall\}$ on the side-branches in code $(T)$ can be matched by entering $M_{a}$ in the right-hand side process $M$. In case that the implementation contains a side-branch with the symbol $\forall$, the specification $M$ will enter the state 
$M_{\forall}$ and require that two branches with labels 1 and 2 follow, however, from definition of $\operatorname{code}(T)$ this is clearly satisfied. A full proof is given in the appendix.

\subsection{The Reduction-Part 2}

We now proceed with the construction of the right-hand side process R. Its implementations should be the codes of all incorrect or rejecting computation trees. To cover the notion of incorrect computation, we define a so-called bad path (see page 6 for definition of the relation Comp).

Definition 3.9. A sequence

$$
c_{1} c_{2} c_{3} c_{4} c_{5} \underbrace{a_{1} a_{2} \ldots a_{n-6} a_{n-5}}_{n-5 \text { elements from } \Xi} d_{1} d_{2} d_{3} d_{4} d_{5}
$$

is called $a$ bad path if $\left(c_{1}, c_{2}, c_{3}, c_{4}, c_{5}, d_{1}, d_{2}, d_{3}, d_{4}, d_{5}\right) \in \Xi^{10} \backslash$ Comp.

To cover the incorrect or rejecting computations, we loop in the process $R$ under all actions, including the auxiliary ones, except for $\mathrm{q}_{\mathrm{acc}}$. For convenience we denote $\Xi^{\prime}=\Xi \cup\{\pi, \sigma\}$. For any bad path, the process $R$ can at any time nondeterministically guess the beginning of its first quintuple, realize it, then perform $n-5$ times a sequence of $\pi$ and $\sigma$, and finally realize the second quintuple. Moreover, we have to allow all possible detours of newly created branches to end in the state $U$ where all available actions from $\Xi^{\prime}$ are always enabled and hence the continuation of any implementation is modally refined by $U$. Formally, for any $\left(c_{1}, c_{2}, c_{3}, c_{4}, c_{5}, d_{1}, d_{2}, d_{3}, d_{4}, d_{5}\right) \in \Xi^{10} \backslash$ Comp we add (disjointly) the following fragment into the process $R$ (see also Fig. 4).

$$
\begin{aligned}
& \mathrm{R} \stackrel{\pi}{\rightarrow} \mathrm{V}_{1} \\
& V_{j} \stackrel{\pi}{\longrightarrow} W_{j} \stackrel{\sigma}{\longrightarrow} V_{j+1} \quad \text { for } 1 \leq j<n+5 \\
& V_{j} \stackrel{c_{j}}{\longrightarrow} X_{j} \quad \text { for } 1 \leq j \leq 5 \\
& V_{n+j} \stackrel{d_{n+j}}{\longrightarrow} X_{5+j} \quad \text { for } 1 \leq j \leq 5 \\
& \mathrm{~V}_{\mathrm{j}} \stackrel{\mathrm{x}}{\rightarrow} \mathrm{U}, \mathrm{W}_{\mathrm{j}} \stackrel{\mathrm{x}}{\rightarrow} \mathrm{U}, \mathrm{V}_{\mathrm{n}+5} \stackrel{\mathrm{x}}{\rightarrow} \mathrm{U} \text { for } 1 \leq \mathrm{j}<\mathrm{n}+5 \text { and } x \in \Xi^{\prime} \\
& \mathrm{U} \stackrel{\mathrm{x}}{-\rightarrow} \mathrm{U} \quad \text { for all } x \in \Xi^{\prime} \\
& \mathrm{R} \stackrel{\mathrm{x}}{-\rightarrow} \mathrm{R} \quad \text { for all } x \in \Xi^{\prime} \backslash\left\{\mathrm{q}_{\mathrm{acc}}\right\}
\end{aligned}
$$

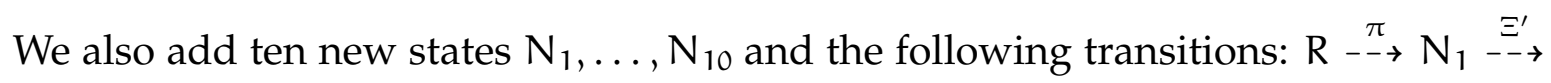
$N_{2} \stackrel{\Xi^{\prime}}{\stackrel{-}{\rightarrow}} N_{3} \stackrel{\Xi^{\prime}}{\rightarrow} N_{4} \stackrel{\Xi^{\prime}}{-\rightarrow} \ldots \stackrel{\Xi^{\prime}}{\rightarrow} N_{10}$ and $N_{1} \stackrel{\text { qace }}{\longrightarrow} N_{10}$ where any transition labelled by $\Xi^{\prime}$ is the abbreviation for a number of transitions under all actions from $\Xi^{\prime}$. 


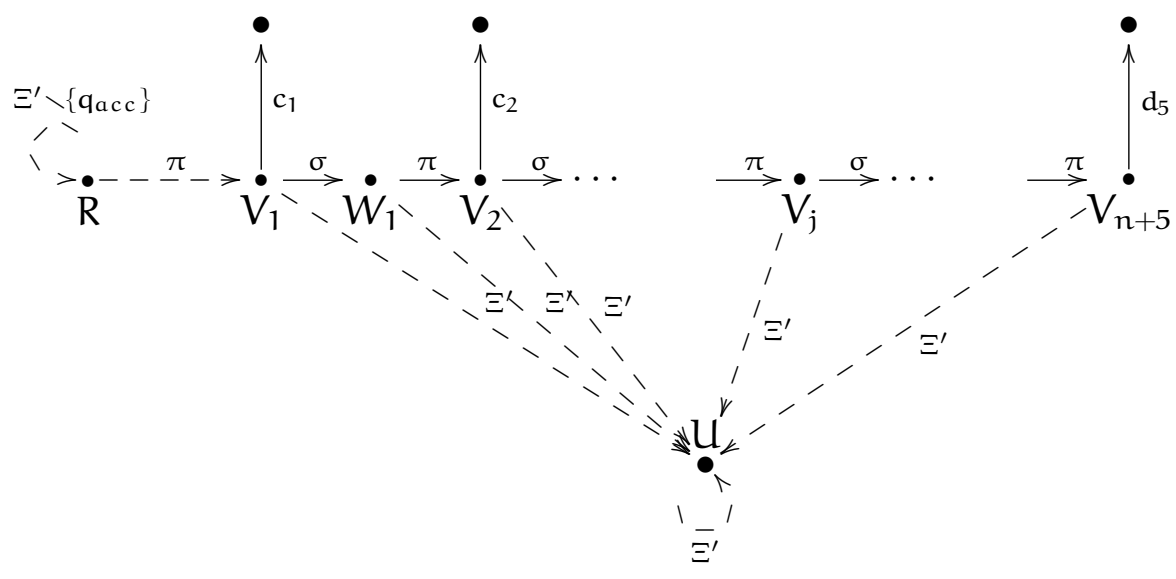

Figure 4: A fragment of the system $R$ for a bad path $c_{1} c_{2} c_{3} c_{4} c_{5} \ldots d_{1} d_{2} d_{3} d_{4} d_{5}$

Remark 3.10. We do not draw these newly added states $\mathrm{N}_{1}, \ldots, \mathrm{N}_{10}$ into Fig. 4 in order not to obstruct its readability. The reason why these states are added is purely technical. It is possible that there is an incorrect computation that ends with the last symbol $\mathrm{q}_{\mathrm{acc}}$ but it cannot be detected by any bad path as defined in Definition 3.9 because that requires (in some situations) that there should be present at least four other subsequent symbols. By adding these new states into the process $\mathrm{R}$, we guarantee that such situations where a branch in a computation tree ends in $\mathrm{q}_{\mathrm{acc}}$ without at least four additional symbols will be easily matched in $\mathrm{R}$ by entering the state $\mathrm{N}_{1}$.

Lemma 3.11. Let I be a tree implementation of $\mathrm{L}$ such that every occurrence of $\mathrm{q}_{\mathrm{acc}}$ in $\mathrm{I}$ is either preceded by a code of a bad path or does not continue with the encoding of at least four other symbols. Then $\mathrm{I} \leq_{m} \mathrm{R}$.

Proof sketch. All branches in I that do not contain $\mathrm{q}_{\mathrm{acc}}$ can be easily matched by looping in $R$ and all branches that contain an error (bad path) before $q_{a c c}$ appears on that branch are matched by entering the corresponding state $V_{1}$ and at some point ending in the state U which now allows an arbitrary continuation of the implementation I (including the occurrence of the state $\mathrm{q}_{\mathrm{acc}}$ ). A full proof is given in the appendix.

Remark 3.12. Lemma 3.11 demonstrates the point where we need our special encoding of configurations using the alternation of $\pi$ and $\sigma$ actions together with side-branches to represent the symbols in the configurations. If the configurations were encoded directly as sequences of symbols on a linear path, the construction would not work. Indeed, the must path of alternating $\sigma$ and $\pi$ actions in the process $\mathrm{R}$ is necessary to ensure that the bad path entered in the left-hand 


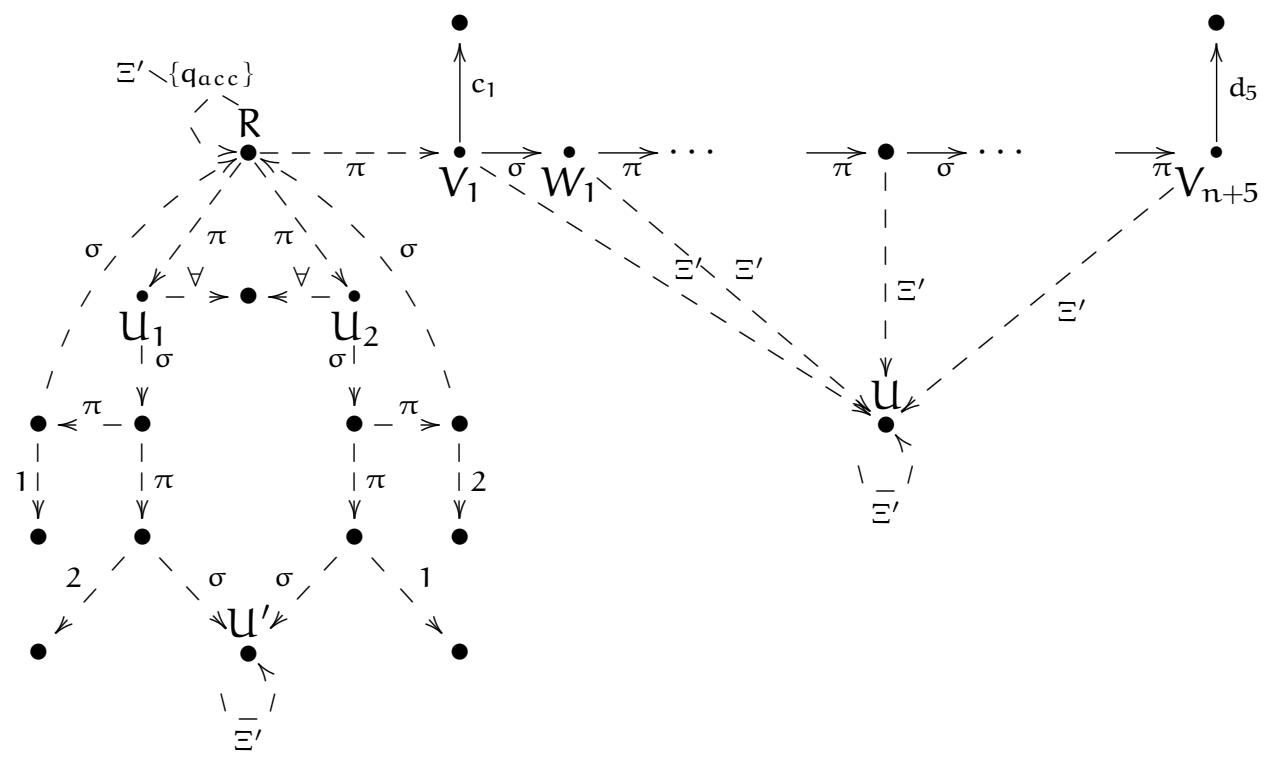

Figure 5: Full specification of the process $R$

side implementation I is indeed realizable. This path cannot be replaced by a linear path of must transitions containing directly the symbols of the configurations because the sequence of $n-5$ symbols in the middle of the bad sequence would require exponentially large MTS to capture all such possible sequences explicitly and the reduction would not be polynomial.

Let us now finish the definition of the process R. Note that in ALBA even rejecting computation trees can still contain several correct computation paths ending in accepting configurations. We can only assume that during any universal branching in a rejecting tree, at least one of the two possible successors forms a rejecting branch. The process $R$ must so have the possibility to discard the possibly correct computation branch in universal branching and it suffices to make sure that the computation will continue with only one of the branches.

So in order to finish the construction of $R$ we add an additional fragment to $R$ as depicted in Fig. 5 (it is the part below $R$ that starts with branching to $U_{1}$ and $U_{2}$ ).

The construction of the process $R$ is now finished (recall that the part of the construction going from $\mathrm{R}$ to the right is repeated for any bad path of the machine $\mathcal{M}$ ). Because the newly added part of the construction does not use any must transitions, it does not restrict the set of implementations and hence Lemma 3.11 still holds. The following two lemmas show that the added part of the construction correctly handles the universal branching. 
Lemma 3.13. Let I be a tree implementation of $\mathrm{L}$ which is not, even after removing any of its branches, a code of any accepting computation tree of $\mathcal{M}$ on the input $w$. Then $\mathrm{I} \leq_{m} R$.

Proof sketch. We should prove that in the universal branching in I, the specification $\mathrm{R}$ can choose one of the two possible continuations and discard the checking of the other one. This is achieved by entering either the state $U_{1}$ or $U_{2}$ whenever the next side-branch in I contains the symbol $\forall$. From $U_{1}$ the continuation under the second successor is discarded by entering the state $U^{\prime}$ and symmetrically from $U_{2}$ the continuation under the first successor is discarded. We argued in Lemma 3.11 for the rest. A full proof is given in the appendix.

Lemma 3.14. Let $\mathrm{T}$ be an accepting computation tree of an $A L B A \mathcal{M}$ on the input $w$. Then $\operatorname{code}(\mathrm{T}) \not \underline{m}_{\mathrm{m}} \mathrm{R}$.

Proof sketch. Indeed, in code $(\mathrm{T})$ any branch ends in a configuration containing $\mathrm{q}_{\mathrm{acc}}$ and there is no error (bad path), so the specification R clearly does not have code $(T)$ as its implementation. A full proof is given in the appendix.

\subsection{Summary}

We can now combine the facts about the constructed systems $L$ and $R$ in the following theorem.

Theorem 3.15. An $A L B A \mathcal{M}$ accepts an input $w$ iff $\mathrm{L} \not \mathbb{L}_{\mathrm{t}} \mathrm{R}$.

Proof. If $\mathcal{M}$ accepts the input $w$ then clearly it has an accepting computation tree T. By Lemma $3.8 \operatorname{code}(T) \leq_{m} L$ and by Lemma $3.14 \operatorname{code}(T) \not_{m} R$. This implies that $L \not_{t} R$.

On the other hand, if $\mathcal{M}$ does not accept $w$ then none of the tree implementations of L represents a code of an accepting computation tree of $\mathcal{M}$ on $w$. By Lemma 3.13 this means that any tree I such that $I \leq_{m} L$ satisfies that $I \leq_{m} R$ and hence $L \leq_{t t} R$ which is by Lemma 3.5 equivalent to $L \leq_{t} R$.

Corollary 3.16. The problem of checking thorough refinement on finite modal transition systems is EXPTIME-hard.

In fact, we can strengthen the result by adapting the above described reduction to the situation where the left-hand side system is of a fixed size.

Theorem 3.17. The problem of checking thorough refinement on finite modal transition systems is EXPTIME-hard even if the left-hand side system is fixed.

Proof. The proof is in the appendix. 


\section{Thorough Refinement Is in EXPTIME}

In this section we provide a direct algorithm for deciding thorough refinement between MTSs in EXPTIME. Given two processes A and B over some finite-state MTSs, the algorithm will decide if there exists an implementation $I$ that implements $A$ but not $B$, i.e. $I \leq_{m} A$ and $I \underline{Z}_{m}$ B.

For a modal transition systems $B$, we introduce the syntactical notation $\bar{B}$ to denote the semantical complement of B, i.e. $\mathrm{I} \leq_{m} \overline{\mathrm{B}}$ iff $\mathrm{I} \not \underline{\leq}_{m}$ B. Our algorithm now essentially checks for consistency (existence of a common implementation) between $A$ and $\bar{B}$ with the outcome that they are consistent if and only if $A \not \underline{t}_{t} B$.

In general, we shall check for consistency of sets of the form $\left\{A, \bar{B}_{1}, \ldots, \bar{B}_{k}\right\}$ in the sense of existence of an implementation I such that $\mathrm{I} \leq_{m} A$ but $I \mathbb{E}_{m} B_{i}$ for all $i \in$ $\{1, \ldots, k\}$. Before the full definition is given, let us get some intuition by considering the case of consistency of a simple pair $A, \bar{B}$. During the arguments, we shall use CCS-like constructs (summation and action-prefixing) for defining implementations.

Clearly, if for some $B^{\prime}$ with $B \stackrel{a}{\longrightarrow} B^{\prime}$ and for all $A_{i}$ with $A \stackrel{a}{\longrightarrow} A_{i}$ we can find an implementation $I_{i}$ implementing $A_{i}$ but not $B^{\prime}$ (i.e. we demonstrate consistency between all the pairs $\left.A_{i}, \overline{B^{\prime}}\right)$, we can claim consistency between $A$ and $\bar{B}$ : as a common implementation I simply take $\mathrm{H}+\sum_{i} a \cdot I_{i}$, where $H$ is some arbitrary implementation of $A$ with all a-derivatives removed.

We may also conclude consistency of $A$ and $\bar{B}$, if for some $A^{\prime}$ with $A \stackrel{a}{-\rightarrow} A^{\prime}$, we can find an implementation $I^{\prime}$ of $A^{\prime}$, which is not an implementation of any $B^{\prime}$ where $B \stackrel{a}{-\rightarrow}$ $B^{\prime}$. Here a common implementation would simply be $\mathrm{H}+\mathrm{a} \cdot \mathrm{I}^{\prime}$ where $\mathrm{H}$ is an arbitrary implementation of $A$. However, in this case we will need to determine consistency of the set $\left\{A^{\prime}\right\} \cup\left\{\overline{B^{\prime}} \mid B \stackrel{a}{-} \rightarrow B^{\prime}\right\}$ which is in general not a simple pair.

Definition 4.1. Let $M=(P,-\rightarrow, \longrightarrow)$ be an MTS over the action alphabet $\Sigma$. The set of consistent sets of the form $\left\{A, \bar{B}_{1}, \ldots, \bar{B}_{k}\right\}$, where $A, B_{1}, \ldots, B_{k} \in P$, is the smallest set Con such that $\left\{\mathrm{A}, \bar{B}_{1}, \ldots, \bar{B}_{k}\right\} \in$ Con whenever $\mathrm{k}=0$ or for some $\mathrm{a} \in \Sigma$ and some $\mathrm{J} \subseteq\{1, \ldots, k\}$, where for all $\mathrm{j} \in \mathrm{J}$ there exists $\mathrm{B}_{j}^{\prime}$ such that $\mathrm{B}_{j} \stackrel{\mathrm{a}}{\longrightarrow} \mathrm{B}_{\mathbf{j}}^{\prime}$, we have

1. $\left\{\mathrm{A}^{\prime}, \overline{\mathrm{B}_{j}^{\prime}} \mid j \in J\right\} \in$ Con for all $\mathrm{A}^{\prime}$ with $\mathrm{A} \stackrel{\mathrm{a}}{\longrightarrow} \mathrm{A}^{\prime}$, and

2. $\left\{\mathrm{A}_{\ell}, \overline{\mathrm{B}_{\ell}^{\prime}} \mid \mathrm{B}_{\ell-\rightarrow} \stackrel{\mathrm{a}}{\rightarrow} \mathrm{B}_{\ell}^{\prime}\right\} \cup\left\{\overline{\mathrm{B}_{j}^{\prime}} \mid j \in \mathrm{J}\right\} \in$ Con for all $\ell \notin \mathrm{J}$ and some $\mathrm{A}_{\ell}$ with $\mathrm{A} \stackrel{\mathrm{a}}{\rightarrow} \mathrm{A}_{\ell}$.

Lemma 4.2. Given processes $A, B_{1}, \ldots, B_{k}$ of some finite $M T S$, there exists an implementation I such that $\mathrm{I} \leq_{m} A$ and $\mathrm{I} \not_{m} B_{i}$ for all $i \in\{1, \ldots, k\}$ if and only if $\left\{A, \bar{B}_{1}, \ldots, \bar{B}_{k}\right\} \in$ Con. 
Computing the collection of consistent sets $\left\{A, \bar{B}_{1}, \ldots, \bar{B}_{k}\right\}$ over an MTS $(\mathrm{P},-\rightarrow, \longrightarrow)$ may be done as a simple (least) fixed-point computation. The running time is polynomial in the number of potential sets of the form $\left\{A, \bar{B}_{1}, \ldots, \bar{B}_{k}\right\}$ where $A, B_{1}, \ldots, B_{k} \in P$, hence it is exponential in the number of states of the underlying MTS. This gives an EXPTIME algorithm to check for thorough refinement.

Theorem 4.3. The problem of checking thorough refinement on finite modal transition systems is decidable in EXPTIME.

Example 4.4. Consider S and T from Fig. 1. We have already mentioned in Section 2 that $\mathrm{S} \leq_{\mathrm{t}} \mathrm{T}$. To see this, we will attempt (and fail) to demonstrate consistency of $\{\mathrm{S}, \overline{\mathrm{T}}\}$ according to Definition 4.1, which essentially asks for a finite tableau to be constructed. Now, in order for $\{\mathrm{S}, \overline{\mathrm{T}}\}$ to be concluded consistent, we have to establish consistency of $\left\{\mathrm{S}_{1}, \overline{\mathrm{T}}_{1}, \overline{\mathrm{T}}_{2}\right\}$ - as $\mathrm{T}$ has no must-transitions the only choice for $\mathrm{J}$ is $\mathrm{J}=\emptyset$. Now, to establish consistency of $\left\{\mathrm{S}_{1}, \overline{\mathrm{T}}_{1}, \overline{\mathrm{T}}_{2}\right\}$ both $\mathrm{J}=\emptyset$ and $\mathrm{J}=\{1\}$ are possibilities. However, in both cases the requirement will be that $\{\mathrm{S}, \overline{\mathrm{T}}\}$ must be consistent. Given this cyclic dependency together with the minimal fixed-point definition of Con it follows that $\{\mathrm{S}, \overline{\mathrm{T}}\}$ is not consistent, and hence that $\mathrm{S} \leq_{\mathrm{t}} \mathrm{T}$.

Example 4.5. Consider $\mathrm{S}$ and $\mathrm{U}$ from Fig. 1 . Here $\mathrm{S} \not_{\mathrm{t}} \mathrm{U}$ clearly with $\mathrm{I}=a .0$ as a witness implementation. Let us demonstrate consistency of $\{\mathrm{S}, \overline{\mathrm{U}}\}$. Choosing $\mathrm{J}=\emptyset$, this will follow from the consistency of $\left\{\mathrm{S}_{1}, \overline{\mathrm{U}}_{1}\right\}$. To conclude this, note that $\mathrm{J}=\{1\}$ will leave us with the empty collection of sets-as $S_{1}$ has no must-transitions-all of which are obviously consistent.

Note that in the case of $B$ being deterministic, we only need to consider pairs of the form $\{A, \bar{B}\}$ for determining consistency. This results in a polynomial time algorithm (see also [5] for an alternative proof of this fact). Similarly, if the process B is of a constant size, our algorithm runs in polynomial time as well.

Corollary 4.6. The problem of checking thorough refinement between a given finite modal transition system and a finite deterministic or fixed-size modal transition system is in P.

To conclude, by Theorem 4.3 and Corollary 3.16 we get our main result.

Theorem 4.7. The problem of checking thorough refinement on finite modal transition systems is EXPTIME-complete. 


\section{Conclusion}

We proved that the problem of checking the thorough refinement relation between two finite-state modal transition systems is EXPTIME-complete. This result completes related complexity results achieved in [3] as the thorough refinement relations on both modal and mixed (where the must transition relation is not necessarily included in the may transition relation) specifications, the common implementation problems on modal and mixed specifications, as well as the consistency problem on mixed specifications are now all EXPTIME-complete. Our EXPTIME-hardness result is proved by reduction from the acceptance problem for alternating linear bounded automata because the problems of consistency and common implementation mentioned above did not seem to provide a suitable starting problem for the reduction.

The fact that the thorough refinement relation is computationally hard means that the relation of modal refinement is more suitable for practical purposes, even though it describes a less desirable notion of syntactic refinement. On the other hand, much of the recent work in the area focuses to a large extend on deterministic specifications $[12,11]$ and here the two notions of refinement coincide. A detailed study of computational complexity of problems on deterministic modal transition systems is provided in [5].

\section{References}

[1] A. Antonik, M. Huth, K.G. Larsen, U. Nyman, and A. Wasowski. 20 years of modal and mixed specifications. Bulletin of the EATCS 95, pages 94-129, 2008.

[2] A. Antonik, M. Huth, K.G. Larsen, U. Nyman, and A. Wasowski. Complexity of decision problems for mixed and modal specifications. In Proc. of FOSSACS'08, volume 4962 of LNCS, pages 112-126. Springer, 2008.

[3] A. Antonik, M. Huth, K.G. Larsen, U. Nyman, and A. Wasowski. EXPTIMEcomplete decision problems for mixed and modal specifications. In Proc. of EXPRESS'08, July 2008.

[4] J. L. Balcazar, J. Gabarró, and M. Santha. Deciding bisimilarity is P-complete. Formal aspects of computing, 4(6 A):638-648, 1992.

[5] N. Beneš, J. Křetínský, K.G. Larsen, and J. Srba. On determinism in modal transition systems. Theoretical Computer Science, 2008. To appear. 
[6] N. Bertrand, S. Pinchinat, and J.-B. Raclet. Refinement and consistency of timed modal specifications. In Proc. of LATA'09, volume 5457 of LNCS, pages 152-163. Springer, 2009.

[7] N. D'Ippolito, D. Fischbein, M. Chechik, and S. Uchitel. MTSA: The modal transition system analyser. In Proc. of ASE'08, pages 475-476. IEEE, 2008.

[8] N. D'Ippolito, D. Fischbein, H. Foster, and S. Uchitel. MTSA: Eclipse support for modal transition systems construction, analysis and elaboration. In Proc. of (ETX'07), pages 6-10. ACM, 2007.

[9] H. Fecher and H. Schmidt. Comparing disjunctive modal transition systems with an one-selecting variant. J. of Logic and Alg. Program., 77(1-2):20-39, 2008.

[10] P. Godefroid, M. Huth, and R. Jagadeesan. Abstraction-based model checking using modal transition systems. In Proc. CONCUR'01, volume 2154 of LNCS, pages 426-440. Springer, 2001.

[11] T. A. Henzinger and J. Sifakis. The discipline of embedded systems design. IEEE Computer, 40(10):32-40, 2007.

[12] T.A. Henzinger and J. Sifakis. The embedded systems design challenge. In Proceedings of the 14th International Symposium on Formal Methods (FM'06), volume 4085 of LNCS, pages 1-15. Springer-Verlag, 2006.

[13] M. Huth, R. Jagadeesan, and D.A. Schmidt. Modal transition systems: A foundation for three-valued program analysis. In Proc. of ESOP'01, volume 2028 of LNCS, pages 155-169. Springer, 2001.

[14] K.G. Larsen, U. Nyman, and A. Wasowski. On modal refinement and consistency. In Proc. of CONCUR'07, volume 4703 of LNCS, pages 105-119. Springer, 2007.

[15] K.G. Larsen and B. Thomsen. A modal process logic. In Proc. of LICS'88, pages 203-210. IEEE, 1988.

[16] S. Nanz, F. Nielson, and H.R. Nielson. Modal abstractions of concurrent behaviour. In Proc. of SAS'08, volume 5079 of LNCS, pages 159-173. Springer, 2008.

[17] J.-B. Raclet. Residual for component specifications. In Proc. of the 4th International Workshop on Formal Aspects of Component Software, 2007. 
[18] J.-B. Raclet, E. Badouel, A. Benveniste, B. Caillaud, and R. Passerone. Why are modalities good for interface theories? In Proc. of $A C S D^{\prime} 09,2009$. To appear.

[19] Z. Sawa and P. Jančar. Behavioural equivalences on finite-state systems are PTIMEhard. Computing and informatics, 24(5):513-528, 2005.

[20] M. Sipser. Introduction to the Theory of Computation. Course Technology, 2006.

[21] S. Uchitel and M. Chechik. Merging partial behavioural models. In Proc. of FSE'04, pages 43-52. ACM, 2004.

[22] O. Wei, A. Gurfinkel, and M. Chechik. Mixed transition systems revisited. In Proc. of VMCAI'09, volume 5403 of LNCS, pages 349-365. Springer, 2009. 


\section{Appendix}

For convenient argumentations in this appendix we extend the standard game-theoretic characterization of bisimilarity. to the game characterization of modal refinement. This will be often used in the proofs of lemmas to follow.

A modal refinement game (or simply a modal game) on a pair of processes $(\mathrm{S}, \mathrm{T})$ is a twoplayer game between Attacker and Defender. The game is played in rounds. In each round the players change the current pair of processes $(A, B)$ (initially $A=S$ and $B=T$ ) according to the following rule:

1. Attacker chooses an action $a \in \Sigma$ and one of the processes $A$ or $B$. If he chose $A$ then he performs a move $A \stackrel{a}{\rightarrow-\rightarrow} A^{\prime}$ for some $A^{\prime}$; if he chose $B$ then he performs a move $\mathrm{B} \stackrel{\mathrm{a}}{\longrightarrow} \mathrm{B}^{\prime}$ for some $\mathrm{B}^{\prime}$.

2. Defender responds by choosing a transition under $a$ in the other process. If Attacker chose the move from $A$, Defender has to answer by a move $B \stackrel{a}{-\rightarrow} B^{\prime}$ for some $B^{\prime}$; if Attacker chose the move from B, Defender has to answer by a move $A \stackrel{a}{\longrightarrow} A^{\prime}$ for some $A^{\prime}$.

3. The new current pair of processes becomes $\left(A^{\prime}, B^{\prime}\right)$ and the game continues with a next round.

The game is similar to standard bisimulation games with the exception that Attacker is only allowed to attack on the left-hand side under may transitions (and Defender answers by may transitions on the other side), while on the right-hand side Attacker attacks under must transitions (and Defender answers by must transitions in the lefthand side process).

Any play (of the modal game) thus corresponds to a sequence of pairs of processes formed according to the above rule. A play (and the corresponding sequence) is finite iff one of the players gets stuck (cannot make a move). The player who got stuck lost the play and the other player is the winner. If the play is infinite then Defender is the winner.

The following fact is by a standard argument in analogy with strong bisimulation games: $S \leq_{m} T$ iff Defender has a winning strategy in the modal game starting with the pair $(S, T)$; and $S \not_{m} T$ iff Attacker has a winning strategy.

Lemma 3.8 Let $\mathrm{T}$ be a computation tree of an $A L B A \mathcal{M}$ on an input $w$. Then $\operatorname{code}(\mathrm{T}) \leq_{\mathrm{m}} \mathrm{L}$. 
Proof. We shall describe Defender's winning strategy in the modal refinement game starting from the begin node of code $(T)$ and the process L. The tree code $(T)$ clearly begins with the encoding of the initial configuration and an identical part is contained also in the beginning of the process L. Hence the game surely continues from the begin node of the code of the next configuration(s) in T and the process $M$ (otherwise Attacker losses immediately, should he choose any of the side branches). Now Attacker must attack under the action $\pi$ in the left-hand side tree (no must transitions are enabled on the right-hand side from $M$ ) and Defender is matching this move from $M$ in two different ways. Should the Attacker's next state contain a branch with a label a such that $a \in \Xi \backslash\{\forall\}$ then Defender plays $\pi$ and enters the process $M_{a}$ on the right-hand side. In order for Attacker to still have a chance to win, he must play the action $\sigma$ in either of the processes and the players return to the situation where the right-hand side process is again in M. On the other hand if Attacker's next state after playing $\pi$ in the left-hand side process contains a branch with the label $\forall$, then Defender enters under $\pi$ the state $M_{\forall}$. As before, the only reasonable continuation for Attacker is to play $\sigma$ in one of the processes and the players reach a pair of states where the left-hand side process branches under $\pi$ into two different paths (due to Definition 3.6 of code(T)) and the right-hand side process is in the state $M^{\prime}$. Attacker can now choose one of the branches in either of the processes but Defender can safely match such an attack and the players after two rounds return to the situation where the right-hand side is again in the state $M$. To sum up, Defender has a winning strategy and so code $(T) \leq_{m} L$.

Lemma 3.11 Let I be a tree implementation of $\mathrm{L}$ such that every occurrence of $\mathrm{q}_{\mathrm{acc}}$ in $\mathrm{I}$ is either preceded by a code of a bad path or does not continue with the encoding of at least four other symbols. Then $\mathrm{I} \leq_{\mathrm{m}} \mathrm{R}$.

Proof. We synthesize a winning strategy for Defender starting from the root of I and the process $\mathrm{R}$ in order to prove $\mathrm{I} \leq_{\mathrm{m}} \mathrm{R}$.

Note that as long as the right-hand side process is in the state $\mathrm{R}$ then Attacker can attack only from the left-hand side processes. Defender's strategy is as follows:

1. If Attacker attacks under a $\pi$ transition leading to a subtree from which at least one branch begins with the encoding of a bad path, Defender answers by a $\pi$ transition from $\mathrm{R}$ leading to a state $\mathrm{V}_{1}$ representing the fragment in the right-hand side process corresponding to this bad path. After that, Attacker is forced to switch sides and play under the must-transitions the whole sequence of $\sigma$ and $\pi$ actions until 
reaching $\mathrm{V}_{\mathrm{n}+5}$; Defender will match this sequence by following the branch corresponding to this bad path in the left-hand side process and finally win. Should Attacker during this phase at any time decide to play again from the left-hand side, Defender will "escape" immediately to the state $U$ from which Defender has a clear winning strategy.

2. On any other Attacker's move, Defender simply loops in R and Attacker cannot have played the action $q_{\text {acc }}$ yet (the only action disabled in $R$ ) due to the assumption of the lemma. Should Attacker play a $\pi$ transition enabling $q_{\text {acc }}$ such that there is no continuation with at least 9 other transitions (i.e. 4 additional encoded symbols), Defender will enter the state $N_{1}$ (see Remark 3.10) and win.

The above defined strategy is winning for Defender and so I $\leq_{m} R$.

Lemma 3.13 Let $\mathrm{I}$ be a tree implementation of $\mathrm{L}$ which is not, even after removing any of its branches, a code of any accepting computation tree of $\mathcal{M}$ on the input $w$. Then $\mathrm{I} \leq_{m} R$.

Proof. For any tree implementation I $\leq_{m} \mathrm{~L}$, which cannot be pruned to a code of any accepting computation tree, we extend the strategy for Defender from Lemma 3.11. The first rule remains unchanged.

Otherwise, if Attacker chose a $\pi$ transition leading to a state in the left-hand side process with the action $\forall$ enabled, we know (from the definition of the process $L$, see Proposition 3.7 part 2. and 5.) that a $\sigma$ action must follow and then there are at least two branches under $\pi$, one of them enabling the action 1 and the other one the action 2; because I is not an encoding of an accepting computation tree (not even after being pruned out) either (i) all subtrees beginning with the action 1 are either rejecting or incorrect or (ii) all subtrees beginning with the action 2 are either rejecting or incorrect. In case (i) Defender responds by entering $\mathrm{U}_{1}$, in case (ii) by entering $\mathrm{U}_{2}$. After Attacker plays the above mentioned action $\sigma$ followed by one of the $\pi$ actions, Defender responds by the $\sigma$ action leaving (i) $\mathrm{U}_{1}$ or (ii) $\mathrm{U}_{2}$ and then by one of the two $\pi$ actions so that the Attacker's move on the left-hand side is correctly matched. In case (i) if Attacker plays the $\pi$ move with a following branch under 2, or in case (ii) if Attacker plays the $\pi$ move with the following branch under 1 , Defender will aim at entering the process $\mathrm{U}^{\prime}$ and after the following $\sigma$ move wins as any implementation is a refinement of $U^{\prime}$. Hence Attacker is forced to choose, in case (i), some first branch and, in case (ii), some second branch and after the necessary action $\sigma$ the game continues from a configuration where the right-hand side process is again in $R$. 
Finally, if none of the previous cases applies, Defender simply mimics any Attacker's move by looping in R. Note that in this case Attacker cannot have played the action $\mathrm{q}_{\mathrm{acc}}$ because we assume that the implementation I, even after removing any if its branches, does not encode any accepting computation tree.

As the above defined strategy is winning for Defender, we conclude that $I \leq_{m} R$.

Lemma 3.14 Let $\mathrm{T}$ be an accepting computation tree of an $A L B A \mathcal{M}$ on the input $w$. Then $\operatorname{code}(T) \not Z_{m} R$.

Proof. Note that in T every branch ends with a configuration containing the accepting state $\mathrm{q}_{\mathrm{acc}}$. It is so clear that Attacker can easily win by playing repeatedly the transition $\pi$ followed by the transition $\sigma$ in the tree code $(T)$. Defender is forced to stay in the state R because any branch in the tree is correct and hence Defender cannot "escape" by playing the $\pi$ move to the state $V_{1}$ for some bad path (should Defender play like this, Attacker would switch the sides and play the must sequence of $\pi$ and $\sigma$ transitions until Defender is proven to be cheating and Attacker wins).

The only situation when Defender can play a $\pi$ move going to the state $U_{1}$ or $U_{2}$ is when Attacker (in the left-hand side process) is inside a code of a configuration following a universal configuration and after he played $\pi$ the next label is $\forall$. In case that Defender entered $\mathrm{U}_{1}$, Attacker simply continues on the left-hand side by taking the first successor configuration, and in case that Defender entered $\mathrm{U}_{2}$, Attacker chooses the second successor configuration. After the sequence of one $\pi$ and one $\sigma$ move in the left-hand side process, Defender is forced to return to the state $R$ (otherwise Attacker wins by playing the action 1 resp. 2 in the left-hand side process). Eventually, after reaching an accepting leaf configuration in $T$, Attacker will play the action $q_{\text {acc }}$ in the left-hand side process to which Defender has no answer from the process R. As we have described Attacker's winning strategy, we conclude that code $(T) \not_{m} R$.

Theorem 3.17. The problem of checking thorough refinement on finite modal transition systems is EXPTIME-hard even if the left-hand side system is fixed.

Proof. The size of the MTS underlying the process $L$ in the previous construction is clearly dependent on the size of the input ALBA. We will show that the construction can be slightly modified in order to obtain a fixed-size system on the left-hand side of the thorough refinement checking.

Clearly, there are two places in the system for $L$ that are dependent on the size of the original ALBA. First, it is the encoding of the initial configuration (the path from $L$ 


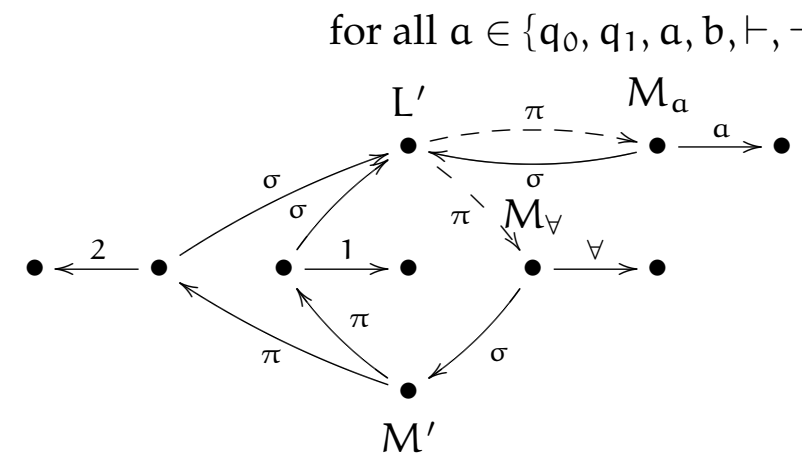

Figure 6: Process L'

to $M$ ) and second, it is the number of loops on $M$ which is dependent on the number of the ALBA's states. The latter case can be dealt with in a straightforward manner, by encoding the states with binary strings of two symbols, say $q_{0}$ and $q_{1}$. This changes the compatible 10-tuples into compatible $\left(2 \cdot\left\lceil\log _{2}|\mathrm{Q}|\right\rceil+8\right)$-tuples, but this change does not affect the construction heavily.

The more interesting part is that of the encoding of the initial configuration. We use a trick similar to that of the previous construction. We change the left-hand side process so that its implementations may exhibit almost arbitrary behaviour. This new process L' is depicted in Fig. 6. Clearly, L' has more implementations than the original process L, namely those that have a branch which starts with something different than the encoding of the initial configuration. Thus, we need to extend the right-hand side process so that it also admits this kind of implementations.

The new process $R^{\prime}$ is built as follows. There are paths encoding the incorrect initial configurations, one for each position of the configuration, i.e. there is a path representing that an error (incorrect symbol in the initial configuration) happens in the first symbol of the configuration, a path representing an error in the second symbol, etc. Similarly to the previous construction we also include may transitions for escaping to universal process, to allow for arbitrary branching in the implementations. To this we furthermore add all transitions the original process $R$ has, including transitions to $R$ itself. Note that this implies that $R \leq_{m} R^{\prime}$.

Similarly to the previous construction, we need to show two things. First, that any implementation of $\mathrm{L}^{\prime}$ that is not an encoding of a correct accepting computation tree even after pruning is an implementation of $R^{\prime}$, and second, that the encoding of a correct accepting computation tree is not an implementation of $\mathrm{R}^{\prime}$. 


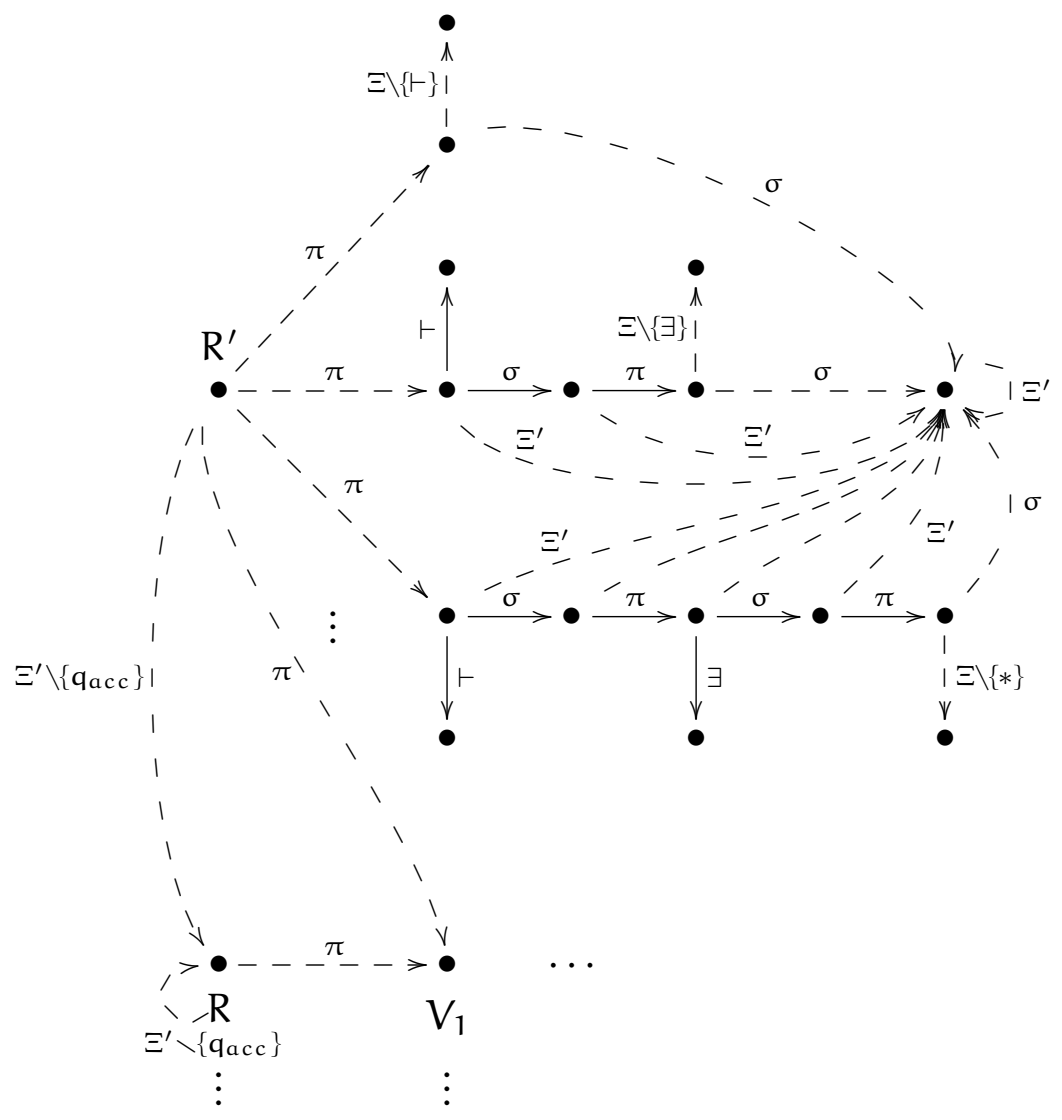

Figure 7: Process R' 
Regarding the first claim let us fix an implementation $I$ of $L^{\prime}$ that is not a correct accepting computation tree. Consider transitions from I lying on branches that do not start with an encoding of the initial configuration. These are matched by the newly created transitions from $R^{\prime}$ to the paths encoding the branches that begin with an error. Let us further consider all transitions from I that lie only on branches beginning with correct encodings of the initial configuration. The corresponding subtrees are then implementations of $R$. But we know that $R \leq_{m} R^{\prime}$, so the whole $I$ is also an implementation of $R^{\prime}$.

The second claim then easily follows from the fact that the encoding of a correct accepting computation tree is neither an implementation of $R$, nor can be matched by any of the bad paths added to $R^{\prime}$, as it clearly has to start with a correct initial configuration. We thus have that the input ALBA accepts the input $w$ if and only if $L^{\prime} \underline{Z}_{t} R^{\prime}$.

Lemma 4.2 Given processes $A, B_{1}, \ldots, B_{k}$ of some finite MTS, there exists an implementation I such that $\mathrm{I} \leq_{m} \mathrm{~A}$ and $\mathrm{I} \mathbb{Z}_{m} \mathrm{~B}_{i}$ for all $i \in\{1, \ldots, k\}$ if and only if $\left\{\mathrm{A}, \overline{\mathrm{B}}_{1}, \ldots, \overline{\mathrm{B}}_{\mathrm{k}}\right\}$ is consistent.

Proof. Because Con is defined as the smallest set, let by Con ${ }_{1}, \operatorname{Con}_{2}, \mathrm{Con}_{3}, \ldots$ denote the nondecreasing sequence of sets according to in which round the elements (consistent sets) where added to Con. So Con ${ }_{1}$ contains exactly all the consistent sets $\left\{A, \overline{B_{1}}, \ldots, \overline{B_{k}}\right\}$ where $k=0$, Con $_{2}$ contains all the consistent sets that were added to Con $_{1}$ in one iteration of the definition, etc.

For the if-direction we prove by induction on $n$ that whenever $\left\{A, \bar{B}_{1}, \ldots, \bar{B}_{k}\right\} \in$ $\operatorname{Con}_{n}$ then there exists an implementation I such that $\mathrm{I} \leq_{m} A$ and $\mathrm{I} \not_{m} B_{i}$ for all $i \in\{1, \ldots, k\}$.

The base case $n=1$ is trivial. For the induction step assume that $\left\{A, \bar{B}_{1}, \ldots, \bar{B}_{k}\right\} \in$ Con $_{n+1}$. Then there exists $a \in \Sigma, J \subseteq\{1, \ldots, k\}$ with fixed $B_{j}^{\prime}$ such that $B_{j} \stackrel{a}{\longrightarrow} B_{j}^{\prime}$ for all $j \in J$ such that

1. $\left\{A^{\prime}, \overline{B_{j}^{\prime}} \mid j \in J\right\} \in$ Con $_{n}$ for all $A^{\prime}$ with $A \stackrel{a}{\longrightarrow} A^{\prime}$, and

2. $\left\{A_{\ell}, \overline{B_{\ell}^{\prime}} \mid B_{\ell} \stackrel{a}{\rightarrow} B_{\ell}^{\prime}\right\} \cup\left\{\overline{B_{j}^{\prime}} \mid j \in J\right\} \in$ Con $_{n}$ for all $\ell \notin J$ and some $A_{\ell}$ with $A \stackrel{a}{A} \rightarrow A_{\ell}$.

From the first clause above, it follows from IH that for all $A^{\prime}$ where $A \stackrel{a}{\longrightarrow} A^{\prime}$, there exists $I_{A^{\prime}}$ such that $I_{A^{\prime}} \leq_{m} A^{\prime}$ and $I_{A^{\prime}} \not_{m} B_{j}^{\prime}$. Similarly, from the second clause above, it follows from $\mathrm{IH}$ that for any $\ell \notin \mathrm{J}$, there exists $\mathrm{I}_{\ell}$ such that $\mathrm{I}_{\ell} \leq_{\mathrm{m}} A_{\ell}$ but $\mathrm{I}_{\ell} \underline{Z}_{\mathrm{m}} \mathrm{B}_{\ell}^{\prime}$ whenever $\mathrm{B}_{\ell} \stackrel{\mathrm{a}}{\rightarrow} \mathrm{B}_{\ell}^{\prime}(\ell \notin \mathrm{J})$ and $\mathrm{I}_{\ell} \not \mathbb{z}_{\mathrm{m}} \mathrm{B}_{j}^{\prime}$ for all $j \in \mathrm{J}$. Now let

$$
I \equiv \Sigma_{A^{\prime} . A \stackrel{a}{\longrightarrow} A^{\prime}} a \cdot I_{A^{\prime}}+\Sigma_{\ell \notin J} a \cdot I_{\ell}+H
$$


where $\mathrm{H}$ is an arbitrary implementation of $A$ with all a-transitions removed. Then it is straightforward to prove that $I \leq_{m} A$ and $I \not_{m} B_{i}$ for all $i \in\{1, \ldots, k\}$.

Let us first establish $I \leq_{m} A$. Assume that $A \stackrel{a}{\longrightarrow} A^{\prime}$, then $I \stackrel{a}{\longrightarrow} I_{A^{\prime}}$ will provide the match. For $I \stackrel{a}{\rightarrow} \rightarrow I_{\ell}$ then $A \stackrel{a}{\rightarrow} \rightarrow A_{\ell}$ provides the match. To see that $I Z_{m} B_{j}$ for $j \in \mathrm{J}$, we note that $\mathrm{B}_{j} \stackrel{\mathrm{a}}{\longrightarrow} \mathrm{B}_{j}^{\prime}$ cannot be matched by either $\mathrm{I} \stackrel{\mathrm{a}}{\longrightarrow} \mathrm{I}_{\mathrm{A}^{\prime}}$ nor $\mathrm{I} \stackrel{\mathrm{a}}{\longrightarrow} \mathrm{I}_{\ell}$. To see that $\mathrm{I} \underset{\mathrm{m}}{\mathrm{m}} \mathrm{B}_{\ell}$ for $\ell \notin \mathrm{J}$, we note that $\mathrm{I} \stackrel{\mathrm{a}}{\rightarrow} \rightarrow \mathrm{I}_{\ell}$ cannot be matched by any may-transition $\mathrm{B}_{\ell-\rightarrow} \rightarrow \mathrm{B}_{\ell}^{\prime}$.

For the only-if-direction we prove by induction on $n$ that whenever there exists an implementation I such that $I \leq_{m} A$ and $I \not_{m}^{n} B_{i}$ for all $i \in\{1, \ldots, k\}$ then $\left\{A, \bar{B}_{1}, \ldots, \bar{B}_{k}\right\} \in$ Con. Here $S \leq_{m}^{n}$ T if either $n=0$ or (i) whenever $S \stackrel{a}{\rightarrow} S^{\prime}$ then $T \stackrel{a}{\rightarrow} T^{\prime}$ and $S^{\prime} \leq_{m}^{n-1} T^{\prime}$ and (ii) whenever $T \stackrel{a}{\longrightarrow} T^{\prime}$ then $S \stackrel{a}{\longrightarrow} S^{\prime}$ and $S^{\prime} \leq_{m}^{n-1} T^{\prime}$. Hence the relation $\leq_{m}^{n}$ is a natural generalization of the classical bisimulation approximants to modal refinement, and clearly (on finite MTS) we have that $S \leq_{m} T$ iff $S \leq_{m}^{n} T$ for all $n$. Let us so proceed with proving the only-if direction.

The base case $n=0$ is trivial as in this case $k=0$ and hence $\left\{A, \bar{B}_{1}, \ldots, \bar{B}_{k}\right\}=\{A\} \in$ Con. For the induction step assume that $I \leq_{m} A$ and $I \not_{m}^{n+1} B_{i}$ for some I.

Now let $J \subseteq\{1, \ldots, k\}$ such that $j \in J$ iff $B_{j} \stackrel{a}{\longrightarrow} B_{j}^{\prime}$ for some $B_{j}^{\prime}$ such that $I^{\prime} \mathbb{E}_{m}^{n} B_{j}^{\prime}$ whenever $\mathrm{I} \stackrel{\mathrm{a}}{\longrightarrow} \mathrm{I}^{\prime}$. Whenever $A \stackrel{\mathrm{a}}{\longrightarrow} A^{\prime}$ then $\mathrm{I} \stackrel{\mathrm{a}}{\longrightarrow} \mathrm{I}^{\prime}$ for some $\mathrm{I}^{\prime}$ with $\mathrm{I}^{\prime} \leq_{\mathrm{m}} A^{\prime}$. It follows from $\mathrm{IH}$ that $\left\{A^{\prime}, \overline{B_{j}^{\prime}} \mid j \in J\right\} \in$ Con whenever $A \stackrel{a}{\longrightarrow} A^{\prime}$.

For $\ell \notin \mathrm{J}$ we must have $\mathrm{I} \stackrel{\mathrm{a}}{\rightarrow} \mathrm{I}_{\ell}$ such that $\mathrm{I}_{\ell} \not \leq_{\mathrm{m}}^{\mathrm{n}} \mathrm{B}_{\ell}^{\prime}$ whenever $\mathrm{B}_{\ell} \stackrel{\mathrm{a}}{\rightarrow} \rightarrow \mathrm{B}_{\ell}^{\prime}$. Since $\mathrm{I} \leq_{\mathrm{m}} A$ it follows that $A \stackrel{a}{\rightarrow} A_{\ell}$ for some $A_{\ell}$ with $I_{\ell} \leq_{m} A_{\ell}$. It follows that $\left\{A_{\ell}, \overline{B_{\ell}^{\prime}} \mid B_{\ell} \stackrel{a}{-\rightarrow}\right.$ $\left.\mathrm{B}_{\ell}^{\prime}\right\} \cup\left\{\overline{\mathrm{B}_{j}^{\prime}} \mid j \in J\right\} \in$ Con.

It follows that the set J provides the evidence required by the definition to conclude that $\left\{\mathrm{A}, \overline{\mathrm{B}}_{1}, \ldots, \overline{\mathrm{B}}_{\mathrm{k}}\right\} \in$ Con. 